\title{
The methionine salvage pathway-involving ADI1 inhibits hepatoma growth by epigenetically altering genes expression via elevating S-adenosylmethionine
}

\author{
Yu-De Chu' ${ }^{1}$, Hsin-Yu Lai ${ }^{1}$, Li-Mei Pai ${ }^{1,2,3,4}$, Ya-Hui Huang ${ }^{1}$, Yang-Hsiang Lin' ${ }^{1}$ Kung-Hao Liang ${ }^{5}$ and Chau-Ting Yeh ${ }^{1,2}$
}

\begin{abstract}
The 5'-methylthioadenosine (MTA) cycle-participating human acireductone dioxygenase 1 (ADI1) has been implicated as a tumor suppressor in prostate cancer, yet its role remains unclear in hepatocellular carcinoma (HCC). Here, we demonstrated a significant reduction of ADI1, either in protein or mRNA level, in HCC tissues. Additionally, higher ADI1 levels were associated with favorable postoperative recurrence-free survival in HCC patients. By altering ADI1 expression in HCC cells, a negative correlation between ADI1 and cell proliferation was observed. Cell-based and xenograft experiments were performed by using cells overexpressing ADI1 mutants carrying mutations at the metalbinding sites (E94A and H133A, respectively), which selectively disrupted differential catalytic steps, resulting in staying or leaving the MTA cycle. The results showed that the growth suppression effect was mediated by accelerating the MTA cycle. A cDNA microarray analysis followed by verification experiments identified that caveolin-1 (CAV1), a growth-promoting protein in HCC, was markedly decreased upon ADI1 overexpression. Suppression of CAV1 expression was mediated by an increase of S-adenosylmethionine (SAMe) level. The methylation status of CAV1 promoter was significantly altered upon ADI1 overexpression. Finally, a genome-wide methylation analysis revealed that ADI1 overexpression altered promoter methylation profiles in a set of cancer-related genes, including CAV1 and genes encoding antisense non-coding RNAs, long non-coding RNAs, and microRNAs, resulting in significant changes of their expression levels. In conclusion, ADI1 expression promoted MTA cycle to increase SAMe levels, which altered genome-wide promoter methylation profiles, resulting in altered gene expression and HCC growth suppression.
\end{abstract}

\section{Introduction}

During the past decades, cancer becomes a leading cause of human death ${ }^{1}$. Among the most common cancer types, hepatocellular carcinoma (HCC) accounts for the third leading cause for cancer-related death ${ }^{2}$. Of all therapeutic modalities, surgical removal of the liver tumorous part remains the most effective treatment ${ }^{2}$.

Correspondence: Chau-Ting Yeh (chautingy@gmail.com)

${ }^{1}$ Liver Research Center, Chang Gung Memorial Hospital, Taoyuan, Taiwan

${ }^{2}$ Molecular Medicine Research Center, Chang Gung University, Taoyuan,

Taiwan

Full list of author information is available at the end of the article.

Edited by $\mathrm{G}$. Calin
However, only a subset of patients in early cancer stage are qualified for surgical resection ${ }^{3}$. Recently, there are emerging therapies such as transcatheter arterial chemoembolization using new embolizing materials and oral targeted drugs, sorafenib for instance, to treat patients with unresectable $\mathrm{HCC}^{4}$. Yet, the responses to these treatments are usually unsatisfactory ${ }^{4}$. Lacking an effective therapeutic strategy justifies the continuous efforts to investigate detail mechanisms of $\mathrm{HCC}$ progression to discover new therapeutic targets.

Previously, we identified the human acireductone dioxygenase (ADI1 or also named Sip-L and MTCBP1) as a hepatic factor serving as an enhancer for hepatitis $\mathrm{C}$

\section{(c) The Author(s) 2019}

(c) Open Access This article is licensed under a Creative Commons Attribution 4.0 International License, which permits use, sharing, adaptation, distribution and reproduction cc) in any medium or format, as long as you give appropriate credit to the original author(s) and the source, provide a link to the Creative Commons license, and indicate if changes were made. The images or other third party material in this article are included in the article's Creative Commons license, unless indicated otherwise in a credit line to the material. If material is not included in the article's Creative Commons license and your intended use is not permitted by statutory regulation or exceeds the permitted use, you will need to obtain permission directly from the copyright holder. To view a copy of this license, visit http://creativecommons.org/licenses/by/4.0/. 
virus (HCV) cell entry ${ }^{5-7}$. The evolutionarily conserved role of ADI1 has been defined and classified as a member of cupin protein family, which is one of the most functionally diverse superfamilies ${ }^{8-11}$. As an acireductone dioxygenase, ADI1 participates in the methionine salvage pathway or the $5^{\prime}$-methylthioadenosine (MTA) cycle and requires $\mathrm{Fe}^{2+}$ metal ion as a cofactor to execute its function in production of 4-methylthio-2-oxobutanate (MTOB), a key step in the pathway ${ }^{12}$. Alternatively, an "off-pathway" proceeds when a $\mathrm{Ni}^{2+}$ is employed to replace $\mathrm{Fe}^{2+}$ ion in ADI1 enzymatic center and thus produces 3-methyl-thio-propionate ${ }^{13}$.

Besides serving as a key enzyme in MTA cycle, ADI1 has been implied as a potential tumor suppressor in several types of cancers according to its declined level in cancerous tissues $^{11,14,15}$. The mechanisms by which ADI1 functions as a tumor suppressor remain varied and elusive. One proposed model suggested ADI1 represses tumor progression via physically interacting with MT1MMP, an oncogenic protein, and thus abrogating the induced autophagy progression ${ }^{11,15}$. Another possible explanation was provided by Oram et al. ${ }^{14}$, where they demonstrated that elevated expression of ADI1 in prostate cancer cells was correlated to a higher apoptotic rate for an unknown reason. Intriguingly, increased apoptosis was also observed when directly supplementing MTOB in the growth media of pancreas carcinoma, breast cancer, and $\mathrm{HCC}$ cell lines ${ }^{16}$. These findings imply an alternative possibility that the tumor suppressive role of ADI1 is contributed from the enzymatic activity to produce MTOB or downstream metabolites in MTA cycle ${ }^{11,14}$.

Here, we aimed to investigate the role of ADI1 in HCC through clinical correlation, cell-based and xenograft experiments, and genome-wide methylation analysis.

\section{Results}

Down-regulation of ADI1 in HCC

As an attempt to investigate the role of ADI1 in HCC, we performed western blot to assess its levels in cancerous and non-cancerous tissues derived from 161 patients. The baseline clinicopathological information of these patients is listed in Supplementary Table S1. Interestingly, a large proportion of patients exhibited significant reduction of ADI1 in the tumorous parts, compared to those of nontumorous parts from the same patients (Fig. 1a). To gain supporting evidence, the GSE14520 dataset was employed to assess whether ADI1 mRNA also down-regulated. Consistently, the $A D I 1$ transcript significantly reduced in the cancerous part, implying down-regulation of ADI1 might originate from low mRNA expression (Fig. 1b).

If ADI1 plays a tumor suppressive role in HCC, a decreased level of it when tumor progressed to a higher grade should be observed. To examine this view, the TCGA database was used. The objects were assorted with traceable tumor grade to stage I-II or III-IV. Analysis of these two groups revealed that, indeed, the ADI1 mRNA level was significantly reduced along with the progression of HCC to a higher grade (Fig. 1c).

Previously, it has been demonstrated that ADI1 both localized in the cytosolic and nuclear compartments ${ }^{7,14}$. However, the subcellular localizations of ADI1 in HCC tissues remain unclear. To address this issue, Immunohistochemistry (IHC) analysis was performed. It was found that ADI1 decreased in the tumorous parts (Fig. 1d), especially in cytoplasm, when compared to that in non-tumorous parts, consistent with the observation in western blot analysis (Fig. 1a). Additionally, it was found that the nuclear ADI1 was presented in the cancerous tissues in 72/161 (44.7\%) and in the non-cancerous tissues in $10 / 161(6.2 \%)$ patients, respectively $(p<0.001)$. Yet, neither the nuclear ADI1 in the cancerous parts nor the nuclear ADI1 in the non-cancerous parts was associated with postoperative HCC recurrence (recurrence-free survivals, $p=0.072$ and 0.664 , respectively).

\section{ADI1 level positively associated with postoperative recurrence-free survival in patients with HCC}

Subsequently, we sought to determine the clinical relevance of ADI1 level in term of postoperative prognosis in HCC patients. Kaplan-Meyer analysis was employed to compare between the subgroups with high and low ADI1 levels (using the mean of ADI1 protein levels as cutoff, after normalized with that of primary hepatocytes) (Supplementary Fig. S1A). The recurrence-free survival was significantly associated with ADI1 protein expression in the non-tumorous $(p=0.013)$ (Fig. 1e, left panel) but not the tumorous parts $(p=0.097)$ (Fig. 1e, middle panel). However, patients with higher ADI1 levels in the tumorous parts still had a better prognosis, albeit not statistically significant. This could be caused by variations of the ADI1 levels in the corresponding non-cancerous parts. Therefore, to decipher the growth regulatory role of ADI1, it was more precise to examine the survival curves stratified by the $\mathrm{T} / \mathrm{N}$ ratio (Fig. 1e, right panel). The result showed that a higher cancerous/non-cancerous ADI1 ratio (in each patient) was associated with a better prognosis.

Consistently, we found a better prognosis in 144 patients with higher $A D I 1$ mRNA levels, while a poorer prognosis in 218 patients with lower ADI1 mRNA levels $(p=0.0375)$, retrieved from the TCGA database (Supplementary Fig. S1B).

\section{Alteration of ADI1 expression affected hepatoma cell proliferation}

To investigate the issue whether ADI1 served as a tumor suppressor in HCC, proliferation rate was assessed in cells with altered ADI1 expression. To mimic the situation of low ADI1 expression in tumorous tissues, the 

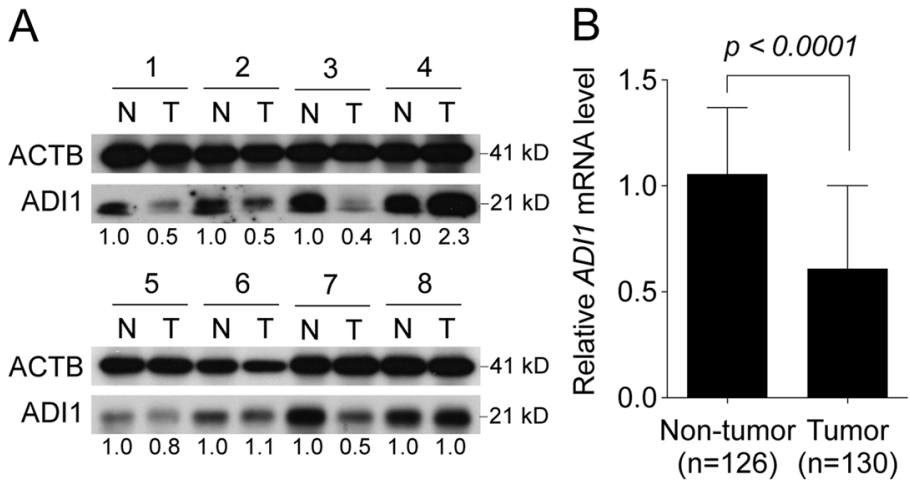

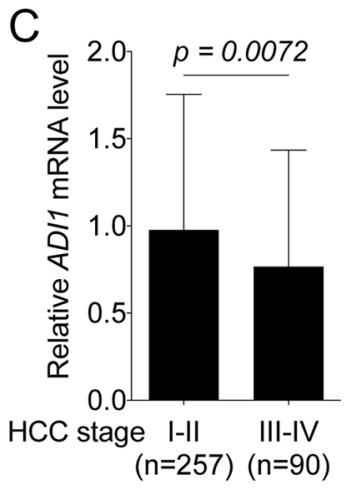

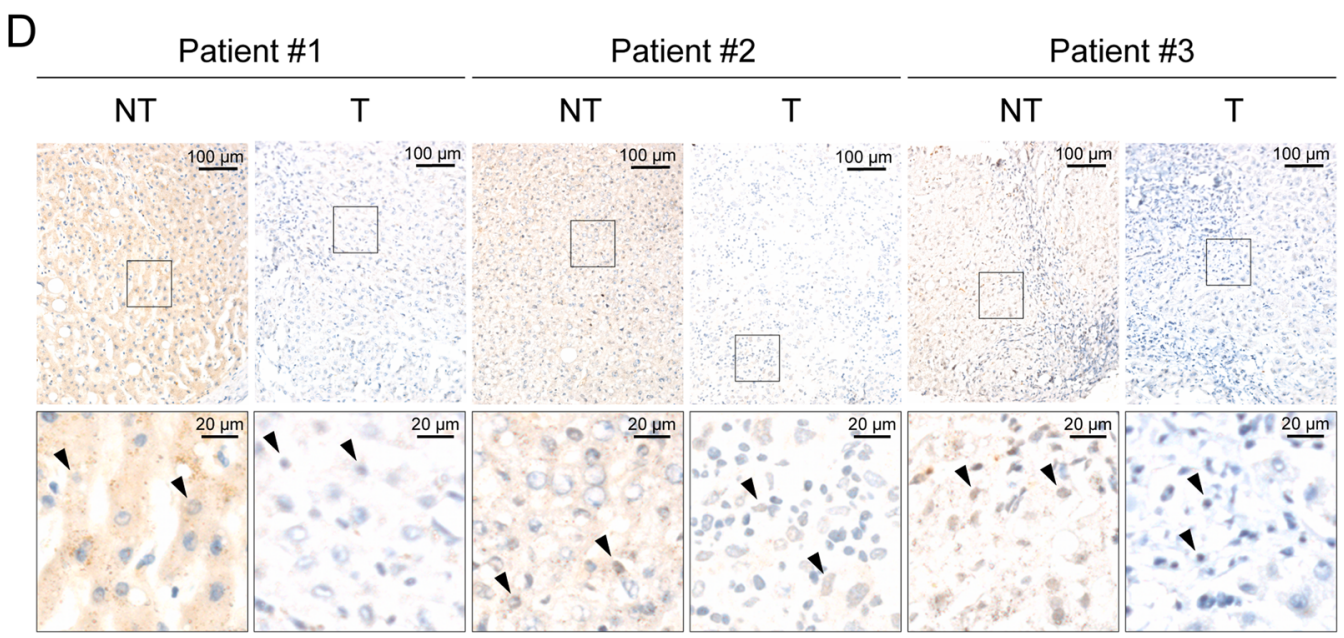

$\mathrm{E}$
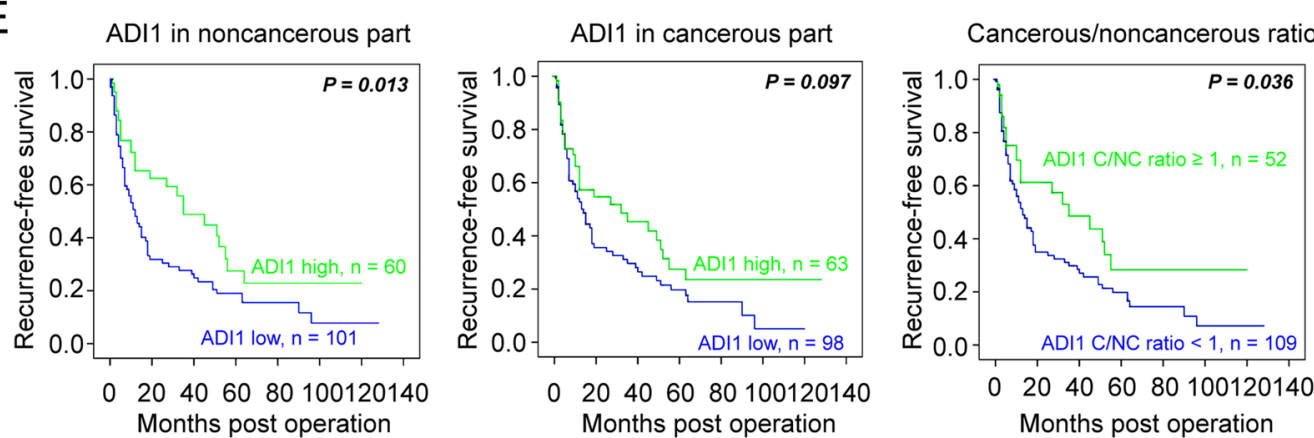

Fig. 1 ADI1 was significantly down-regulated in hepatocellular carcinoma (HCC) tissues and its levels were associated with postoperative prognosis in patients with HCC. a ADI1 levels in tumorous (T) and non-tumorous (N) parts were analyzed by western blotting. ACTB, beta-actin, was used as a loading control. b GSE14520 and c TCGA dataset were utilized to acquire the transcript levels of ADI 1 for cross-reference. The $p$ value was derived by using the unpaired two-tailed Student's t-test. The short vertical bars represented standard deviations. $\mathbf{d} I \mathrm{HC}$ was performed to examine the subcellular distribution of ADI1 in tissues derived from HCC patients. Black arrowheads indicate the positive signal of ADI1 in nuclei. The black line represents the scale bar as indicated. e The protein levels of ADI1 obtained from 161 patients-derived cancerous and non-cancerous tissues were correlated with recurrence-free survival according to the amount in non-tumorous (left), tumorous (middle), and the ratio of tumorous/nontumorous (right) tissues

lentivirus-mediated down-regulation was conducted by using two shRNA clones (Fig. 2a). Notably, we found depletion of ADI1 significantly enhanced cell proliferation (Fig. 2b). Conversely, overexpression of ADI1 led to opposite effects (Fig. 2c-e).
To examine whether the reduced doubling time of ADI1-overexpressing cells was due to the increased apoptosis rate as reported previously ${ }^{14}$, we performed terminal deoxynucleotidyl transferase dUTP nick end labeling (TUNEL) assay to determine the status of cell 


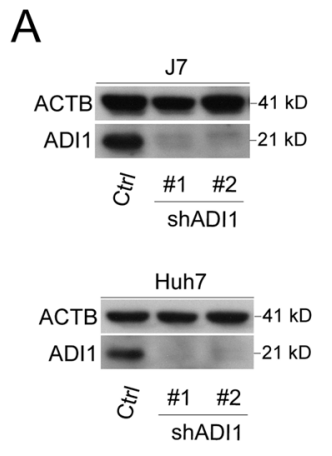

C

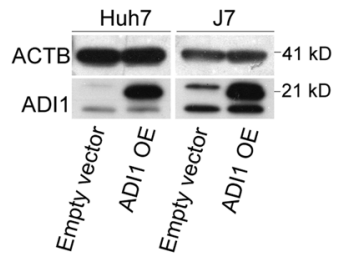

B

$$
\mathrm{J} 7 \text { cell line }
$$

$\rightarrow$ Ctrl shRNA - shADI1 \#1 $₫$ shADI1 \#2

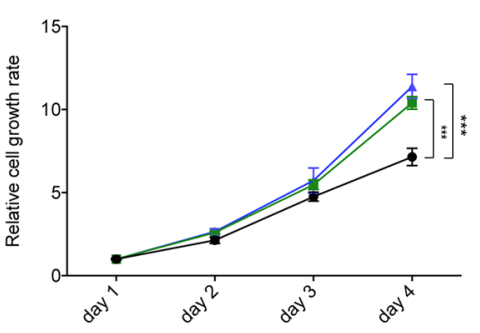

D

J7 cell line

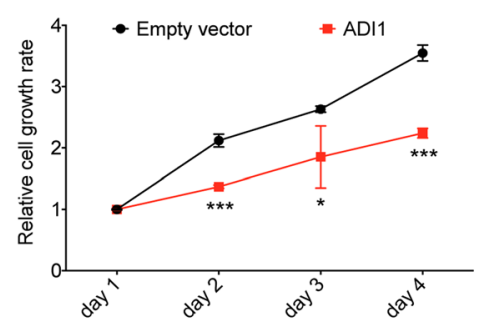

Huh7 cell line

$\rightarrow$ Ctrl shRNA - shADI1\#1 $\approx$ shADI1 \#2

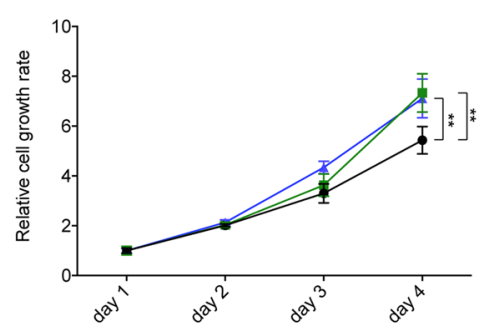

Huh7 cell line

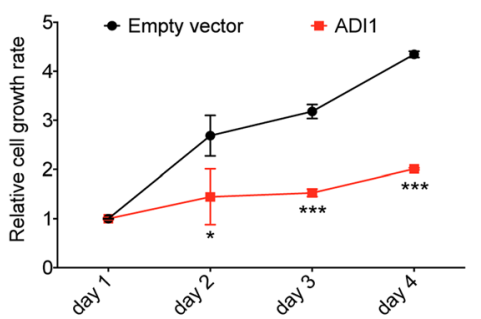

E

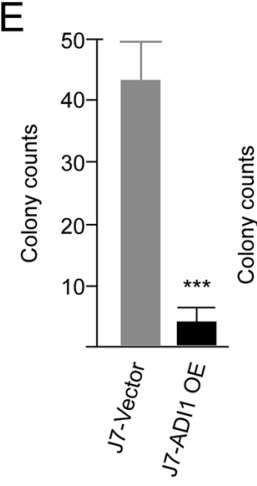

F
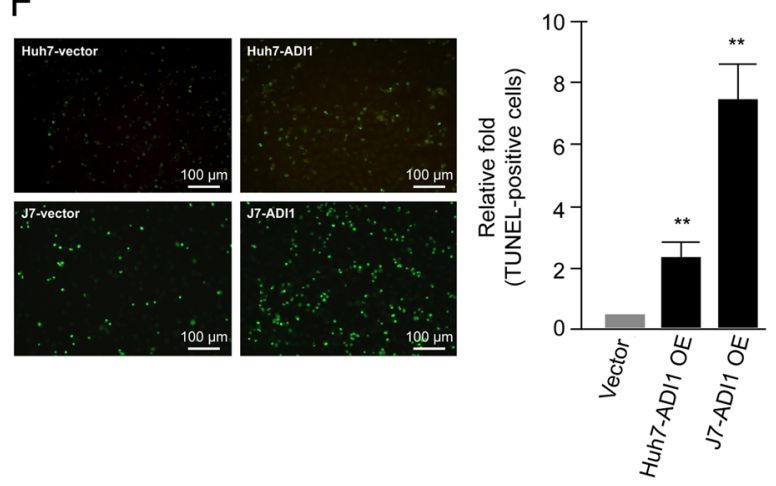

Fig. 2 ADI1 suppressed hepatocellular carcinoma (HCC) cell proliferation. a A representative western blot analysis of samples derived from stably ADI1-knockdown in J7 (upper) and Huh7 (lower) cells. Two targets (\#1 and \#2) for shADI1-mediated knockdown were performed. b The proliferation rates were determined by measuring the florescence of metabolite derived from alarmar blue in $\mathrm{J7}$ (left) or Huh7 (right) cells. Black, mock control (Ctrl); green, shADI1\#1 knockdown; blue, shADI1\#2 knockdown. c Western blots of ADI1 overexpressing (OE) in Huh7 or J7 cells. d Proliferation rates of mock (empty vector, black) or ADI1-overexpressing cells (red) on days 1-4 post seeded to a 96-well plate. e Colony formation assay of ADI1 overexpressing (OE) in $\mathbf{J} 7$ (left) and Huh7 (right) cells. f The representative image of apoptosis status in Huh7 and $\mathrm{J} 7$ cells with or without ADI1 overexpression (OE). The $p$ values were derived by using unpaired two-tailed Student's $t$-test. ${ }^{* *} p<0.01$. The white line represented the scale bar as indicated. The short vertical bars represented standard deviations

apoptosis. Consistently, we found the ratio of apoptotic cells significantly elevated when cells overexpressing ADI1 (Fig. 2f).

\section{The growth suppression effect of ADI1 was mediated through MTA cycle}

The metal-binding ADI1 is well-known for its enzymatic role in MTA cycle ${ }^{12}$. Distinction between the on- or offpathway mediated by ADI1 can be achieved by different ions associated with the enzyme ${ }^{13}$. Key residues for ion binding were identified and validated, such as the E94 for $\mathrm{Ni}^{2+}$ and $\mathrm{H} 133$ for $\mathrm{Fe}^{2+}$ (Fig. 3a) ${ }^{13,14}$. We then attempted to determine whether metal-binding motif was required for ADI1-mediated effect on cell proliferation. As such, Huh7 and J7 cells transfected with ADI1 carrying either wild type or mutant, E94A or H133A mutation (Fig. 3b), were used for examining the effect of growth inhibition. Interestingly, a markedly decrease of cell proliferation was found in cells expressing ADI1 with E94A mutation, but not in the one with mutation at H133A (Fig. 3c, d). 


\section{A}

3-Methylthio-
propionate
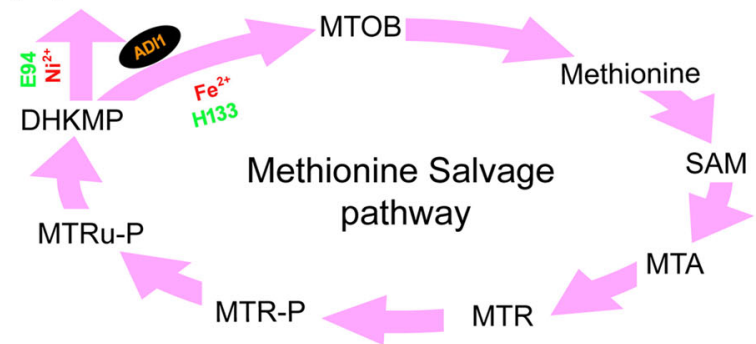
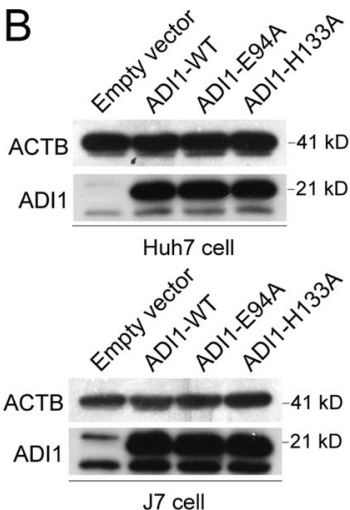

D $\quad$ J cell

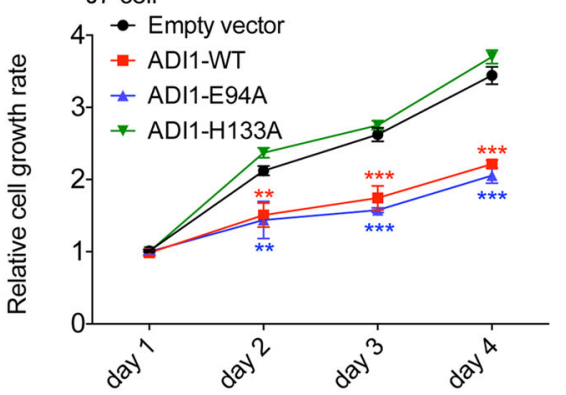

$\partial^{2}+\partial^{2}-\partial^{2} \partial^{2}$

C Huh7 cell
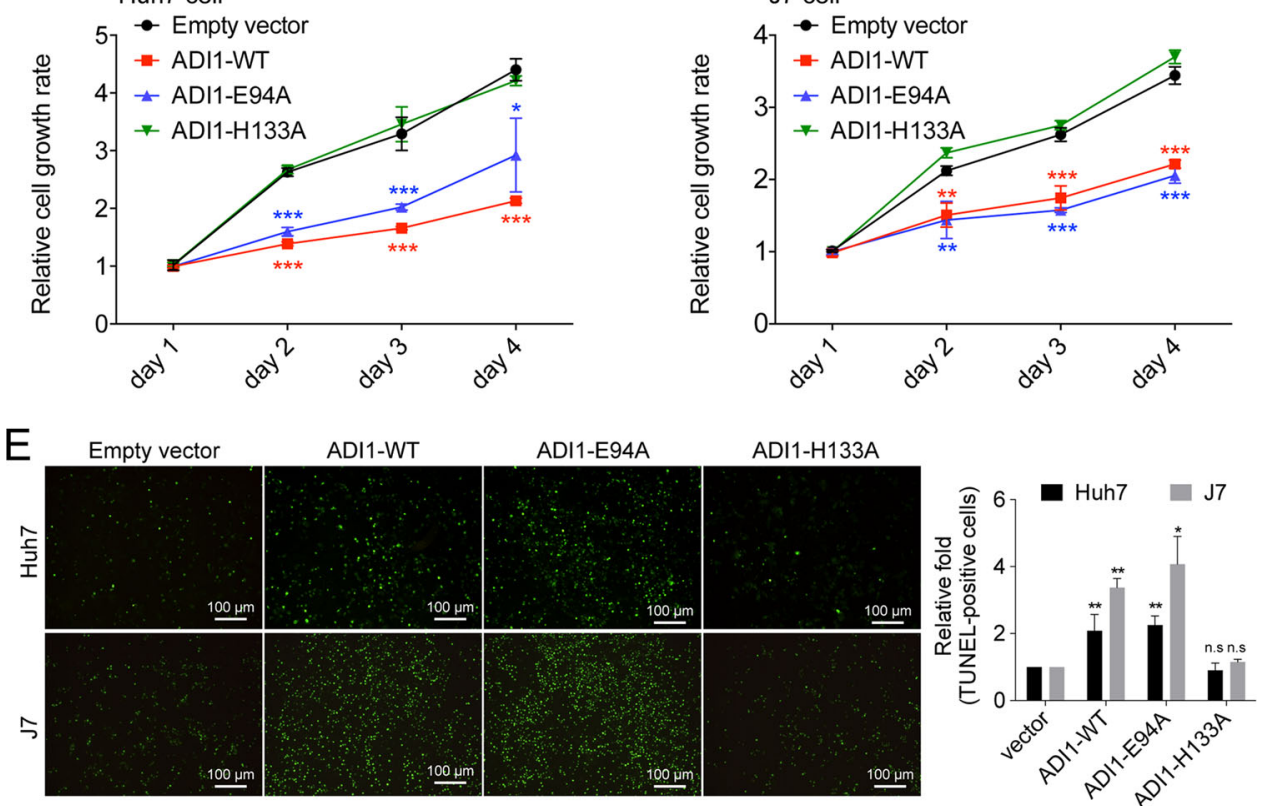

$\mathrm{F}$
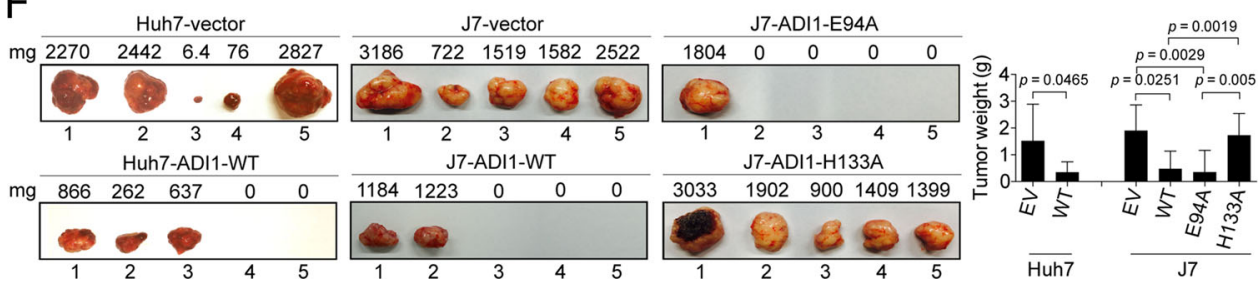

Fig. 3 Growth inhibition effect mediated by ADI1 is through MTA cycle. a Schematic representation of steps in MTA cycle. MTOB, 2-keto-4methylthiobutyrate; SAMe, S-adenosylmethionine; MTA, 5-methyl-thioadenosine; MTR, 5-methyl-thioribose; MTR-P, 5-methyl-thioribose-1-P; MTRu-P, 5-methyl-thioribulose-1-P; DHKMP, 1,2-dihydroxy-3-keto-5-methylthiopentene. b, c Western blot analysis of mutated ADI1 (ADI-H133A and ADI-E94A) stably expressed in Huh7 and $\mathbf{J 7}$ cells. $\mathbf{d}$, e The proliferation rates obtained by measuring the florescence of metabolite derived from alamar blue in Huh7 and $\mathrm{J} 7$ cells. The $p$ value was derived by using unpaired two-tailed Student's $t$-test. ${ }^{* *} p<0.01,{ }^{* * *} p<0.001$. $\mathbf{f}$ Xenograft tumors after 4 weeks of subcutaneous injection into nude mice. Huh7 stably expressing mock control or ADI1-WT were shown (left); J7 cells stably expressing mock control, ADI1-WT, ADI1-E94A or ADI1-H133A, and mock control were shown (right). The $p$ values were derived by using paired one-tailed Student's $t$-test. The short vertical bars represented standard deviations

To examine whether these mutants also altered cell apoptosis as observed in cells carrying wild-type ADI1 (Fig. 2f), additional TUNEL assays were performed. Consistent with the effects of these mutants in cell growth inhibition, it was found that cells expressing ADI1-E94A mutant had an increased percentage of apoptotic cells similar to those expressing wild-type ADI1, but the increment was not found in those expressing H133A 
(Fig. 3e). The results suggested that the growth suppression effects caused by ADI1 and mutants were partly due to their abilities to induce cell apoptosis in HCC cells.

To further confirm the in vitro findings, we performed xenograft experiments. Consistent with our cell-based results, it was found that the tumor volume of xenografts expressing wild-type ADI1 was significantly smaller than that in the control group. Similarly, the xenografts expressing the ADI1-E94A also manifested a reduced capability of tumor growth in nude mice. However, xenografts expressing ADI1-H133A did not display a growth suppression effect (Fig. 3f). These data suggested that the growth suppression effect of ADI1 was mediated through the MTA cycle.

\section{ADI1 inhibited Caveolin-1 (CAV1) expression through MTA cycle to modulate hepatoma cells growth}

Subsequently, to search for downstream effectors, we performed cDNA microarray analysis to identify genes with altered expression levels upon ADI1 overexpression. A subset of dysregulated gene was identified (Fig. 4a). Among them, Caveolin-1 (CAV1) was decreased most dramatically, more than two-folds (Fig. 4a). The caveolae component, CAV1, has been considered as an enhancer of hepatocarcinogenesis due to its ability to promote cell proliferation and invasion through several wellcharacterized HCC-related signaling pathways ${ }^{17}$. To validate the impact of ADI1 on CAV1 expression, western blot analysis and reverse transcriptase quantitative PCR (RT-qPCR) were performed in ADI1 stably expressing cells. As shown in Supplementary Fig. S2A, B, we found the protein and mRNA levels of Caveolin-1 remarkably reduced in ADI1-overexpressing cells. Conversely, knockdown of ADI1 significantly induced CAV1 protein and mRNA expression (Supplementary Fig. S2C, D). To more comprehensively examine the relationship between ADI1 and CAV1, we performed a complementation assay. In this experiment, two independent shRNA clones, \#1 targeted exon yet \#2 recognized the $3^{\prime}$ UTR sequences of $A D I 1$ mRNA, were employed. As a result, we found that \#1 effectively diminished ADI1 expression despite of replenishing it extraneously by transfecting ADIexpressing plasmids in Huh7 and J7 cells (Fig. 4b, and Supplementary Fig. S2E). As anticipated, it was found that the shRNA \#2 also effectively reducing intrinsic ADI1 expression. However, it was unable to reduce the level of extraneous ADI1 since no 3'UTR was present for targeting (Fig. 4b, Supplementary Fig. S2E). Under any condition, CAV1 levels were always negatively regulated by ADI1 (Fig. 4b), indicating that the regulation was not caused by an off-target effect.

According to the results in Fig. 3, we were then curious whether down-regulation of CAV1 was an event downstream of the MTA cycle. Interestingly, we found that only the "on-pathway" ADI1 (wild type or ADI1-E94A) significantly reduced $C A V 1$ expression but not the "offpathway" ADI1-H133A (Fig. 4c, Supplementary Fig. S2F). The IHC analysis of tissues derived from xenografts in Fig. 3f was also in agreement with such notion (Fig. 4d), suggesting the ADI1-mediated suppression of CAV1 was MTA cycle-dependent.

To further examine whether CAV1 was one of the potent downstream effectors in ADI1-mediated repression of cell proliferation, the rescue experiment was performed. As shown in Fig. 4e, f, it was found that the suppressed cell growth could be partially restored by simultaneous expression of CAV1 in HCC cells overexpressed with either wild type or E94A mutated ADI1 (Supplementary Fig. S2F). This observation strongly suggested that the ADI1-CAV1 regulatory axis was required for ADI1-mediated cell growth control.

\section{ADI1 negatively correlated with CAV1 expression in HCC patients}

Next, we investigated whether the negative association between ADI1 and CAV1 levels could be observed in human HCCs. IHC was performed in cancerous and noncancerous liver tissues. As shown in Supplementary Fig. S3A, B, it was found that ADI1 was significantly reduced in tumorous parts, compared to that in adjacent non-tumorous tissues, while a marked reciprocal expression pattern of CAV1 was found in the tumorous and non-tumorous liver tissues, indicating a negative association between these two proteins in human HCCs.

Furthermore, CAV1 mRNA was increased in highergrade HCC tissues compared with lower-grade group in TCGA dataset (Supplementary Fig. S3C). Also, an elevated amount of $C A V 1$ transcript was correlated with poorer clinical outcome, while a lower level predicted better prognosis in HCC patients (Supplementary Fig. S3D). Similar results were observed in GSE14250 dataset (Supplementary Fig. S3E). Further stratification of these patients by ADI1 level revealed that in both cancerous and non-cancerous tissues, a negative association between $A D I 1$ and $C A V 1$ levels was present (Supplementary Fig. S3F).

\section{ADI1-mediated S-adenosylmethionine elevation altered methylation status in CAV1 promoter}

Next, to investigate the underlying mechanism employed by ADI1, we asked whether change of metabolite concentrations in the MTA cycle affected CAV1 expression. Accordingly, we assessed the level of the final product in pathway, $S$-adenosylmethionine (SAMe), upon altered expression of ADI1. As a consequence, it was found that SAMe concentrations were positively associated with the levels of ADI1 (Supplementary Fig. S4A, B). More systemically examination by employing the samples from 

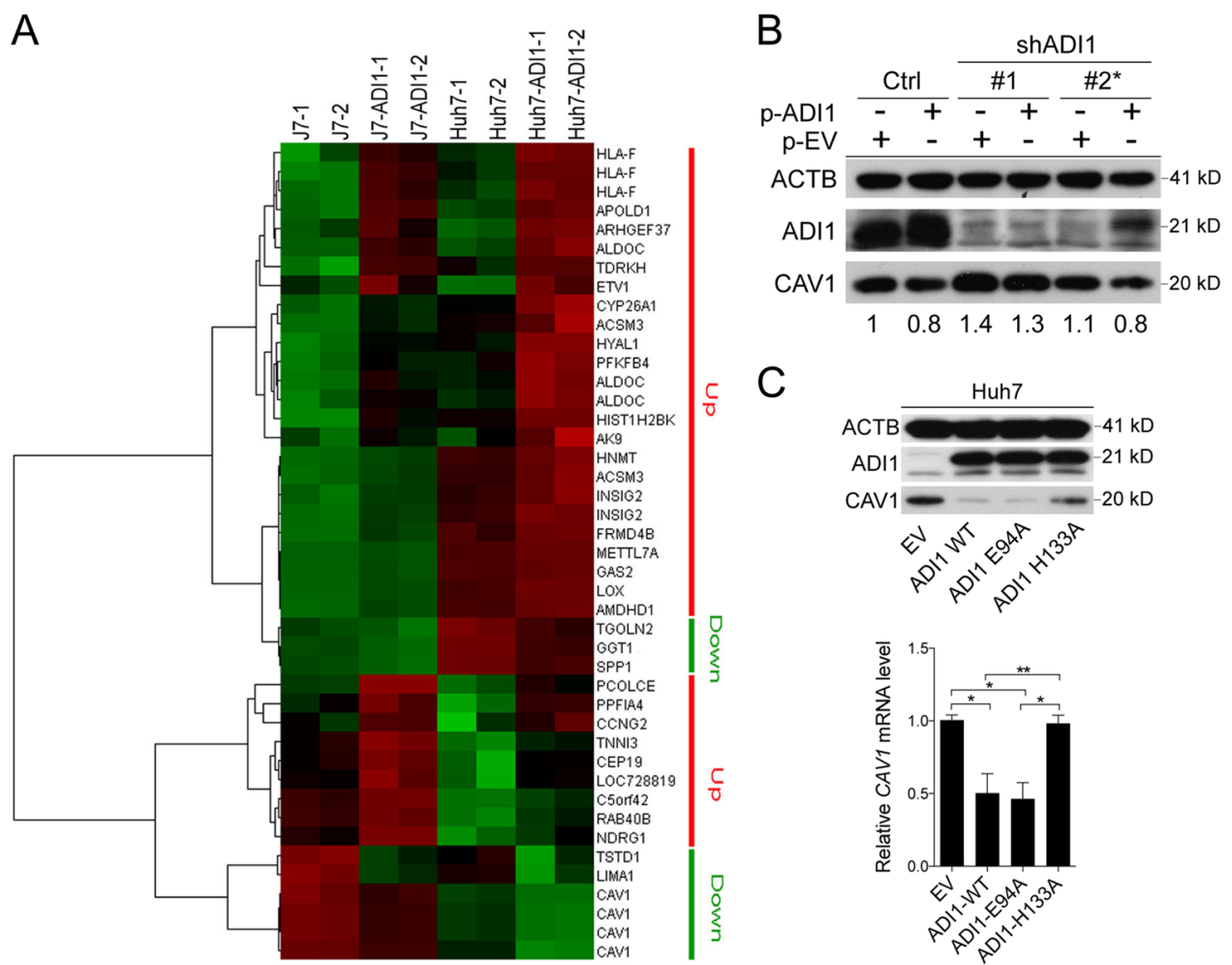

C
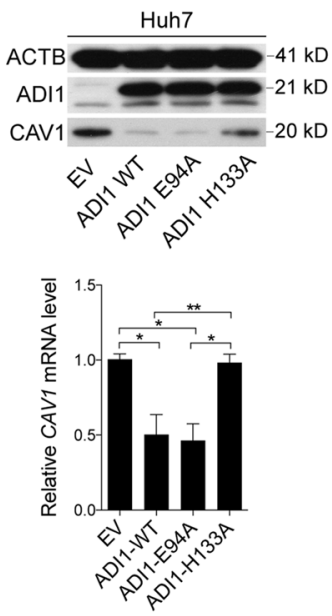

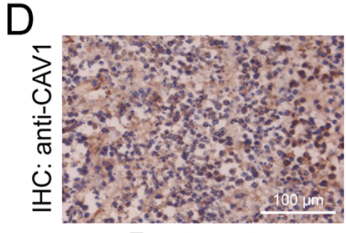

Empty vector

$\mathrm{E}$

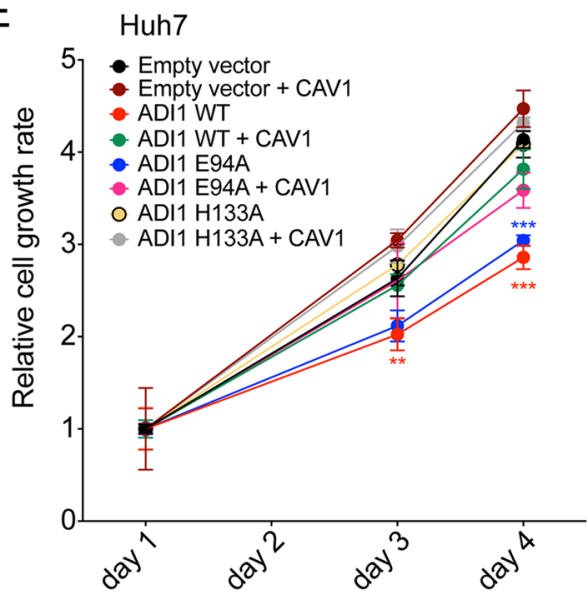

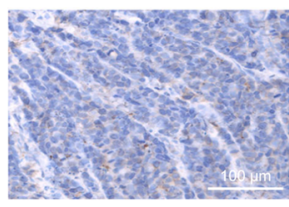

ADI1 WT

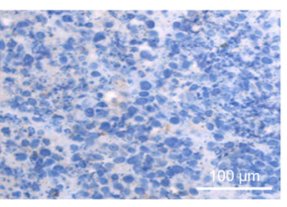

ADI1-E94A

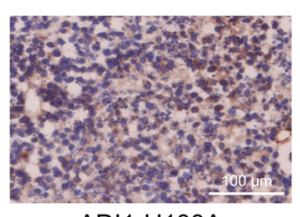

ADI1-H133A

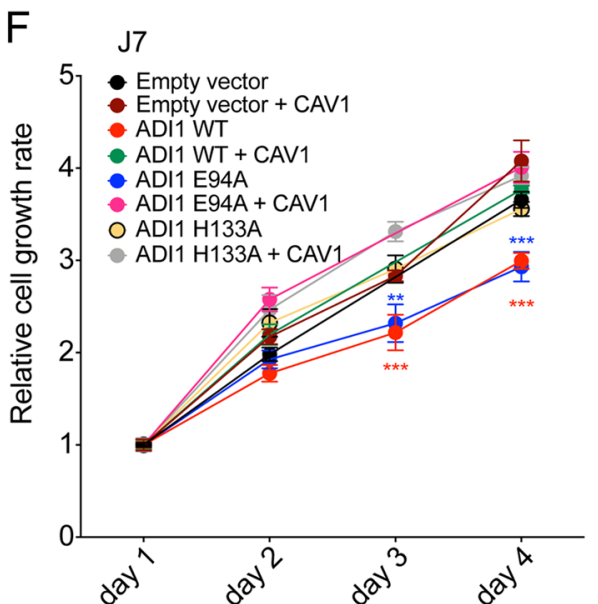

Fig. 4 CAV1 acted as a downstream target of MTA cycle-participating ADI1 in cell proliferation control. a The heat map represented the relative levels of transcripts under overexpression of ADI1 in J7 and Huh7 cells. Two independent cell clones expressing ADI1 ( -1 and -2$)$ were subjected into CDNA array analysis. b Western blot analysis for ADI1 and CAV1 expression levels under shADI1\#1 and \#2 differential target knockdown, with or without extraneous ADI1 complementary expression. p-ADI1, ADI1-expressing plasmid; EV, expressing vector only; ACTB, betaactin, was used as a loading control. c Effect of ADI1 mutants on CAV1 expression in Huh7 cells. Upper panel, western blot; lower panel, relative amounts of CAV1 mRNA obtained by RT-qPCR. ${ }^{* *} p<0.001$. d IHC analysis of CAV1 expression in tissues derived from xenografts (see Fig. 3F). The white line represents the scale bar as indicated. $\mathbf{e}, \mathbf{f}$ The cell proliferation rate was assessed by detecting the florescence of metabolite derived from alarmar blue in Huh7 and J7 cells with indicated treatments. The $p$ value was derived by using unpaired two-tailed Student $t$-test. ${ }^{* *} p<0.01,{ }^{* * *} p<$ 0.001. The short vertical bars represented standard deviations 
complementation assay also exhibited strong correlation between ADI1 and SAMe levels (Fig. 5a). Furthermore, mutation at H133 disrupted the effectiveness of ADI1 in enhancing SAMe synthesis (Fig. 5b).

It is uncertain whether elevation of SAMe concentration had an impact on CAV1 expression. To examine this point, we collected cells and analyzed the CAV1 level following SAMe treatment in various concentrations for $72 \mathrm{~h}$. As shown in Fig. 5c, it was found that the CAV1 protein level decreased in an SAMe dosage-dependent manner. Additionally, it would be interesting to understand if the levels of ADI1 could change in cells treated with distinct SAMe dosages. The results showed that no significant difference of ADI1 levels was found under the indicated SAMe concentrations, suggesting that there was no prominent feedback loop for SAMe to modulate ADI1 expression (Fig. 5c).

Subsequently, owing to the role of SAMe in cellular methylation process, we aimed to investigate whether methylation status of $C A V 1$ promoter was altered when overexpressing ADI1 in HCC cell lines. To achieve this, we isolated genomic DNAs and performed bisulfite conversion. It was then served as a template to amplify the promoter region of $C A V 1$ as previously described ${ }^{18}$. After next-generation sequencing analysis, we found the ratio of several CpG islands, especially the one just one base-pair prior to the transcription start site (TSS), significantly elevated upon ADI1 overexpression either in J7 (Fig. 5d) or Huh7 cells (Supplementary Fig. S4C).

To determine whether the CpG island methylation in the promoter region was important for regulation of CAV1 expression, the inhibitor of DNA methyl transferase (DNMT) was employed in cells with or without ADI1 overexpression. As anticipated, expression of CAV1 was significantly elevated under supplementing DNMT inhibitor, in cells with either wild type or E94A mutated ADI1 overexpression (Fig. 5e, f), confirming the hypothesis that ADI1 increased SAMe and therefore enhanced CAV1 promoter methylation to suppress its expression.

\section{ADI1 modulated promoter methylation status of a subset of genes in HCC cells}

To explore the impact of ADI1 on DNA methylation, we performed methyl-seq analysis and compared the whole-genome methylation status between control and ADI1-overexpressing cells. As a consequence, we identified a large number of sites, which were hyper- or hypomethylated when overexpressing ADI1 (Fig. 6a). Further examination of the genomic features of these sites revealed nearly $15 \%$ of identified sites were located in the gene promoter regions (Fig. 6b). Among these genes, most of them were protein coding genes, including those with declined mRNA observed in cDNA microarray analysis (Fig. 4a), such as TSTD1, LIMA1, and CAV1 (Fig. 6c).
Additionally, the promoter methylation status of some non-coding RNA (ncRNA) genes, including antisense RNAs, long intergenic non-coding RNAs (lincRNAs), and miRNA, were also altered (Fig. 6c). To validate whether ADI1-mediated hypermethylation of these non-coding RNA gene promoter affected their expression, we assessed the expression levels of a panel of genes, which were presumably expressed in hepatic cells, by performing quantitative real-time PCR. Interestingly, we found the expression of some of ncRNA genes actually declined in cells overexpressing ADI1 while increased upon depletion of it (Supplementary Figs. S5-7). By literature searching, we further discovered that some of them were implied as oncogenes in numerous types of cancers, such as TP73AS1 in HCC $^{19}$ (Supplementary Fig. S5), LINC00511 in non-small cell lung cancer ${ }^{20}$ (Supplementary Fig. S6) as well as miR-371 in HCC and pancreatic cancer ${ }^{21,22}$ (Supplementary Fig. S7), supporting our hypothesis that the expression of genes with hyper-methylated promoter might affect gene expression.

Taken together, we demonstrated that ADI1 served as a tumor suppressor in $\mathrm{HCC}$ by modulating productivity of SAMe in MTA cycle. Moreover, a higher amount of SAMe might therefore repress expression of a subset of genes by hypermethylation of their promoters (Fig. 6D).

\section{Discussion}

The human ADI1 has been implicated as a potential tumor suppressor in numerous types of cancers ${ }^{11,14,15,23}$. Here, we demonstrated that ADI1 was significantly reduced, either protein or mRNA level, in HCC. Its expression levels positively correlated with postoperative prognosis of HCC (Fig. 1). These results supported that ADI1 also played a tumor suppressive role in HCC. Moreover, ADI1 expression suppressed HCC cell proliferation (Fig. 2). This could be explained by alteration of apoptosis rate in immortalized hepatic cells when the ADI1 level increased, similar to what was found in prostate cancer (Fig. 2f) ${ }^{14}$. It is appealing to assume that the ability of ADI1 to induce hepatoma cell apoptosis and thus inhibit cell proliferation could be consequences of increasing productivity of MTOB or more downstream metabolites, SAMe for instance, in MTA cycle, as some studies have proposed such possibility ${ }^{16,24,25}$.

Activation of ADI1 enzymatic activity requires metal ion association within the enzymatic center, $\mathrm{Fe}^{2+}$ and $\mathrm{Ni}^{2+}$ for the on- and off-pathway, respectively (Fig. 3a) ${ }^{13}$. The specific amino acid residues responsible for these ions binding were critically identified in previously reports, the E94 and $\mathrm{H} 133$, for $\mathrm{Ni}^{2+}$ and $\mathrm{Fe}^{2+}$, respectively,12,13. Examination of the role of MTA cycle in cancer cells reproduction by mutating these critical residues in ADI1 revealed that the "on-pathway" implemented by $\mathrm{H} 133$ participated in the repression of HCC growth (Fig. 3). It 
A

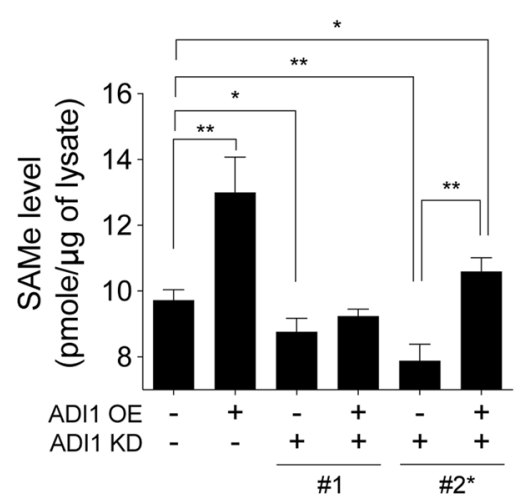

C

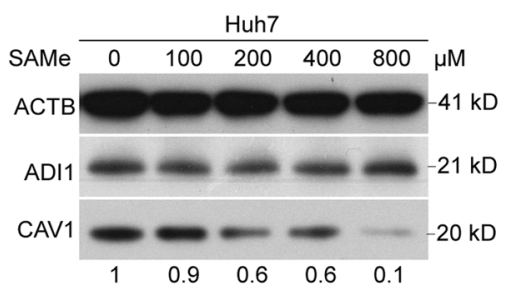

D

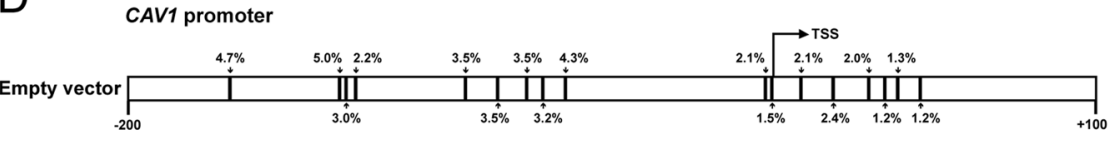

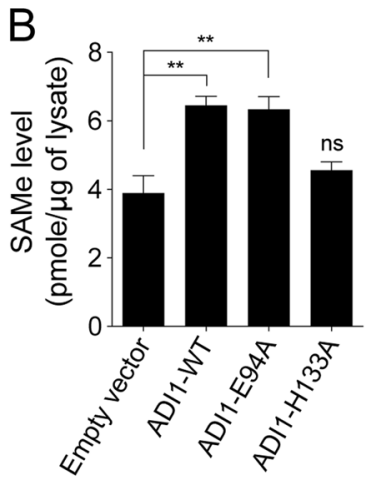

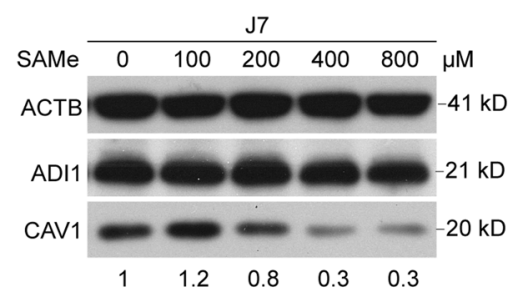

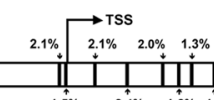

$2.1 \% \quad \underset{1.7 \%}{\longrightarrow} \quad 3.5 \% 1.2 \%$
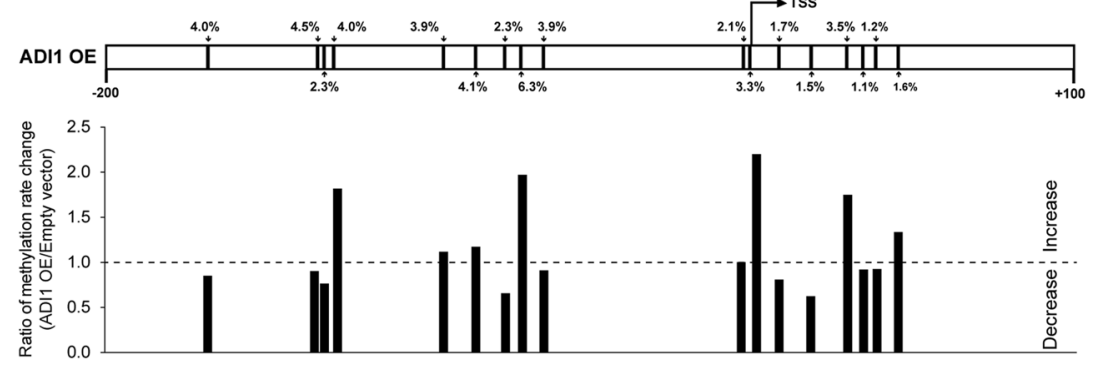

E

F

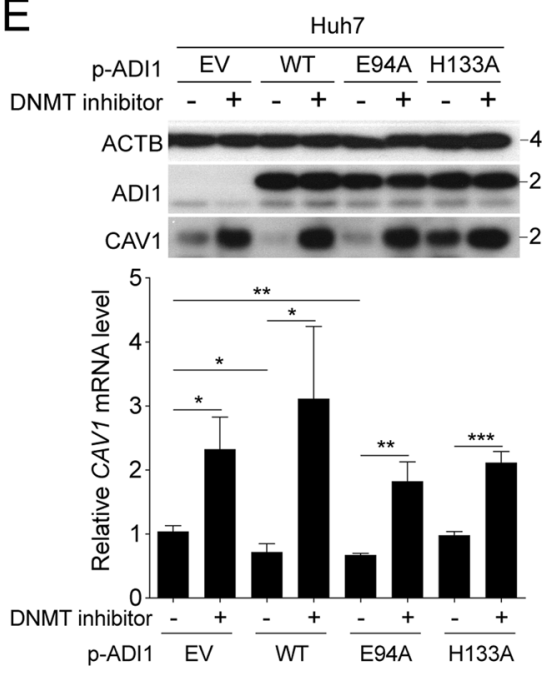

p-ADI1 $\frac{\mathrm{J} 7}{\mathrm{EV}} \frac{\mathrm{WT}}{-++} \frac{\mathrm{E} 94 \mathrm{~A}}{-\quad+} \frac{\mathrm{H} 133 \mathrm{~A}}{-\quad+}$

$-41 \mathrm{kD} \quad \mathrm{ACTB}-41 \mathrm{kD}$

$1 \mathrm{kD} \quad \mathrm{ADl} 1 \mathrm{CO}-21 \mathrm{kD}$

$20 \mathrm{kD}$
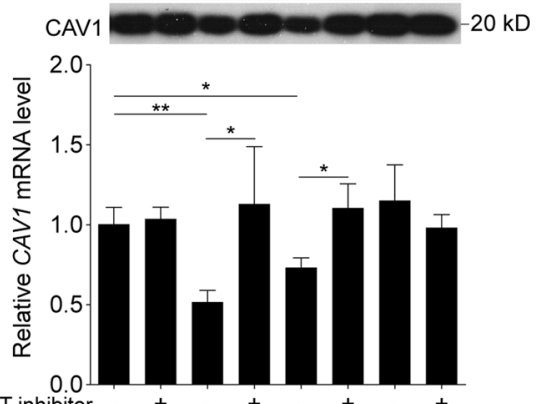

DNMT inhibitor $\frac{-+}{\mathrm{EV}} \frac{-+}{\mathrm{WT}} \frac{-+}{\mathrm{E} 94 \mathrm{~A}} \frac{-+}{\mathrm{H} 133 \mathrm{~A}}$

Fig. 5 (See legend on next page.) 
(see figure on previous page)

Fig. 5 ADI1 increased SAMe levels and suppressed CAV1 expression by altering promoter methylation status. a Quantification of SAMe levels in Huh7 cells with knockdown (KD) or complementary extraneous expression (OE) of ADI1. b Quantification of SAMe levels in Huh7 cells transiently expressing wild type (WT) or mutant ADI1s. $P$ values were calculated by unpaired two-tailed Student's $t$-test. ${ }^{*} p<0.05,{ }^{* *} p<0.01,{ }^{* * *} p<$ 0.001. c Western blot analysis of lysates from Huh7 or J7 cells pre-treated with SAMe in indicated concentrations. $\mathbf{d}$ The bisulfite converted CAV1 promoter region was analyzed by next-generation sequencing in J7 cells with or without ADI1 overexpression (OE). The percentage of methylated CpG islands were labeled. TSS, transcription start site. Western blot and RT-qPCR analysis of samples from empty vector transfected or ADI1expressing e Huh7 or $\mathbf{f} \mathrm{J} 7$ cells pre-treated with or without DNMT inhibitor. The short vertical bars represented standard deviations

remains disputable to date in the literature whether MTA cycle affects cancer progression ${ }^{24,26-29}$. Our results demonstrated that similar to the findings made in prostate cancer, wild-type ADI1 functions in modulating cell apoptosis and growth ${ }^{14}$. However, mutation in H133 or E94 site did not affect ADI1 effects on apoptosis or cell proliferation in LNCaP cells (a prostate cancer cell line), which was quite different from our findings in HCC cell lines, as H133A mutation abolished both functions of ADI1 in cell growth inhibition and apoptosis enhancement (Fig. 3). It is possible that the tumor suppression effect of ADI1 in HCC is mainly manifested through the MTA cycle-mediated increment of SAMe, but might not be so in prostate cancer, because the rate-limiting step for synthesis of SAMe depends on the level of methionine adenosyltransferase in cells. There are two methionine adenosyltransferase isozyme-encoding genes in human chromosome, MAT1A and MAT2A. It is well-known that the gene product of $M A T 1 A$ is a liver-specific protein and is required for SAMe synthesis. Lowering the ratio of the gene products MAT1A/MAT2A has been shown to correlate with a sharp decrease of SAMe level in hepatocytes as well as in HCC cells (for a review, see ref. ${ }^{30}$ ). Further, higher levels of SAMe have been found to induce apoptosis. Taken together, although we could not exclude the possibility that there might be other MTA-cycleindependent pathways involved, the present study strongly suggested that the MTA cycle and/or its downstream metabolite played a major role in ADI1-mediated tumor suppressive effect.

On the other hand, there were a borderline association between postoperative $\mathrm{HCC}$ and nuclear ADI1 $(p=$ 0.072 ) and a higher percentage of nuclear ADI1 in the cancerous tissues $(p<0.001)$. Further investigation should be conducted to clarify the role of nuclear ADI1 in HCC.

During the past decades, a number of preclinical studies have demonstrated the possibility of using SAMe for antiHCC purpose, such as in vitro and in vivo apoptosis enhancement, growth, and angiogenesis inhibition of $\mathrm{HCC}^{25,31-34}$. Furthermore, for clinical usage of SAMe, lines of evidences have demonstrated significantly improvement of hepatic functions in patients with chronic conditions such as cholestasis or alcoholic liver disease $^{35,36}$. Similarly, reduction of alfa-fetoprotein and exhibition of protective capability were also observed under treatment of SAMe in patients with $\mathrm{HCV}$-related cirrhosis and HBV-associated HCC, respectively ${ }^{37,38}$.

SAMe has been well-known for its ability to serve as a methyl donor in methyltransferation and induces methylation on targets ${ }^{39}$. Characterization of substrates for transferring the methyl-group revealed that not merely DNA, but protein and RNA were also capable of methylation ${ }^{40}$. In such case, despite we have observed an elevation of methylation on $\mathrm{CpG}$ island of $C A V 1$ promoter and in promoters of a set of genes from cells overexpressing ADI1, in which SAMe synthesis was enhanced (Figs. 5 and 6), we still cannot exclude the possibility that the mRNA or protein derived from other genes were also methylated and thus affected cell growth.

In summary, our present study has unraveled the role of ADI1 and MTA cycle in suppression of HCC cell proliferation. The level of ADI1 was positively associated with SAMe production and thus impacted on promoter methylation status of a set of genes including the oncogene $C A V 1$. The expression levels of these genes were then altered, leading to cell growth inhibition.

\section{Materials and methods \\ Patients}

Three different cohorts of HCC patients were analyzed. Firstly, 161 paired tumorous and non-tumorous liver tissues used in this study for protein level analysis by western blotting or IHC staining were obtained from Tissue Bank, Chang Gung Memorial Hospital under the permission of the institutional review board, Chang Gung Memorial Hospital, Linkou, Taiwan. Secondly, the online available cDNA microarray dataset, GSE14520, which included the data from 130 tumorous and 126 nontumorous RNA samples, was used to compare the RNA levels of interested genes. Thirdly, transcriptomic results were also retrieved from the TCGA database (https:// genome-cancer.ucsc.edu/), which included 347 RNAsequencing data that derived from defined tumor stages of HCC patients.

\section{IHC staining}

The IHC staining was executed as described previously ${ }^{7}$. The retrieved surgical resected tumorous and 

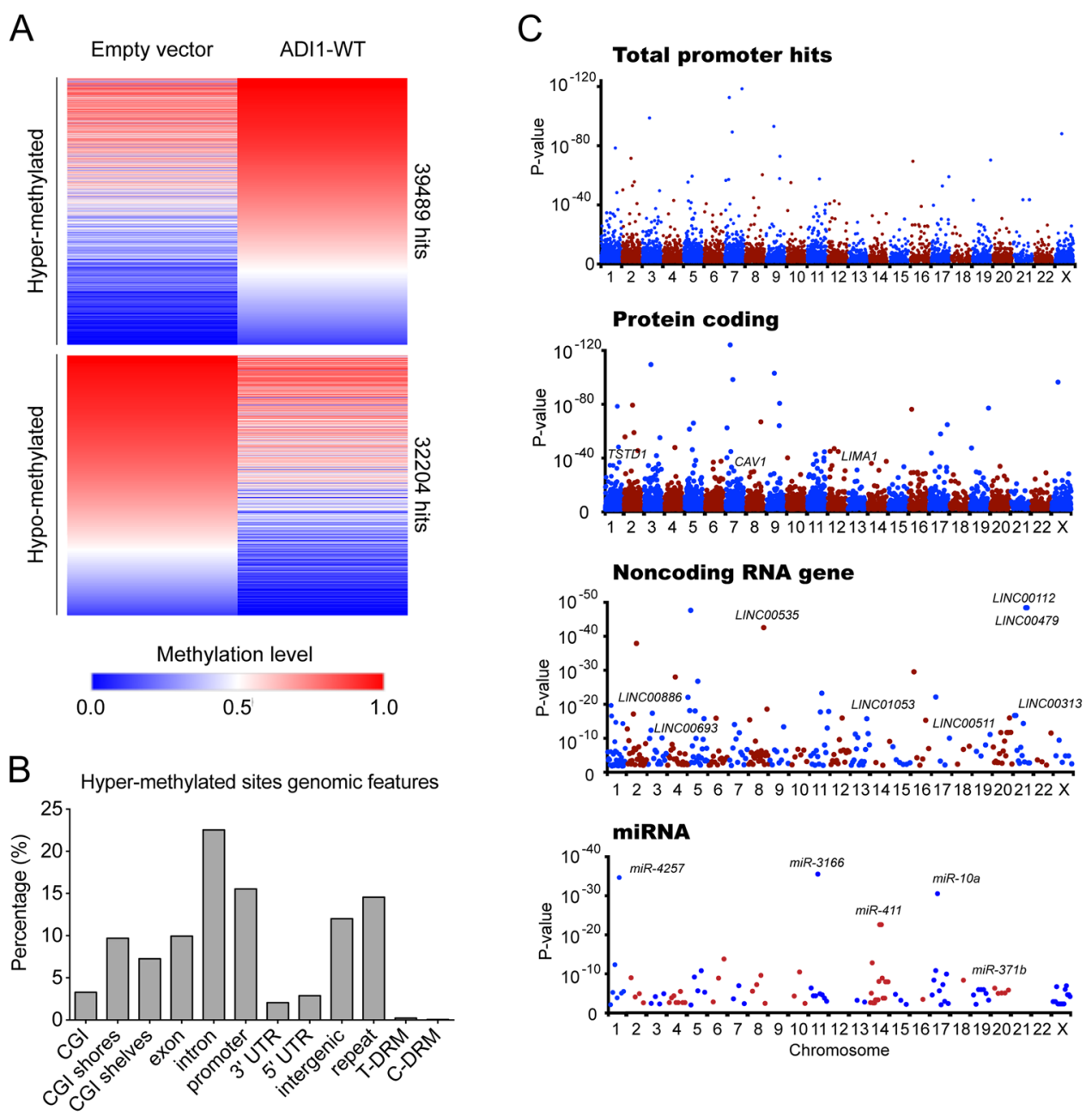

$\mathrm{D}$
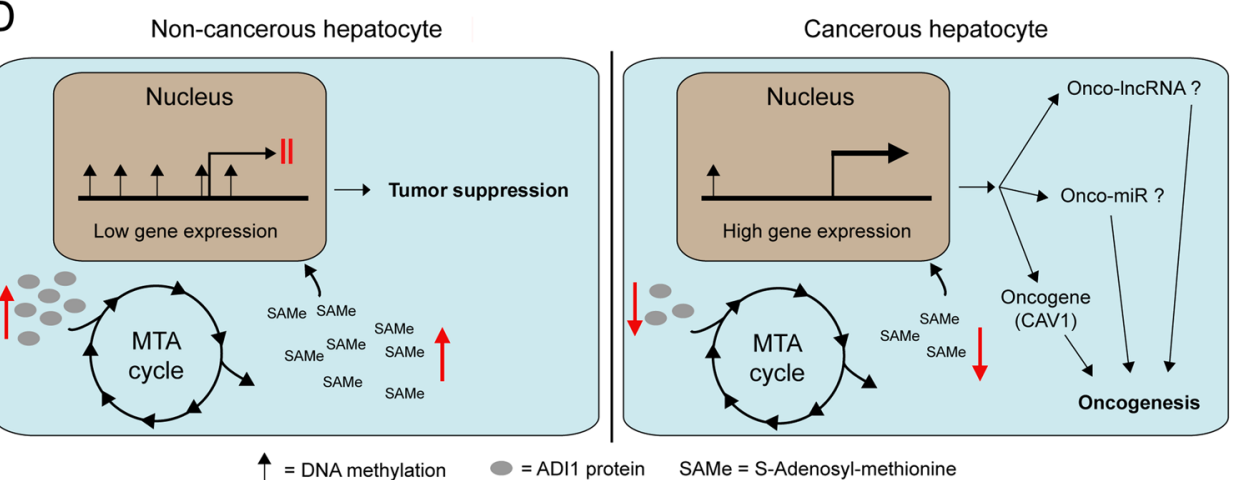

Fig. 6 Promoters of a set of genes were hyper-methylated in ADI1-overexpressing cells. a Heat map representation of DNA methylation levels in $\mathrm{J} 7$ cell with or without ADI1 overexpression. $\mathbf{b}$ The genomic features of hyper-methylated sites in ADI1-overexpressing cells. $\mathbf{c}$ The distributions of significant hypermethylation in total promoters (upper), promoters for protein coding genes (upper middle), promoters for non-coding RNA genes (lower middle), and promoters for miRNA genes (lower). Horizontal axis, chromosome numbers; vertical axis, $p$ value. $\mathbf{d}$ The proposed growth regulatory mechanism obtained from this study. In non-cancerous hepatocytes, ADI (gray oval) levels were high, promoting MTA cycle to generate a large amount of SAMe, which in term modulated genome-wide promoter methylation to achieve a gene expression pattern for tumor suppression. In cancerous hepatocytes, ADI1 was reduced, leading to reduction of SAMe concentrations and thus alterations of genome methylation pattern. As a result, several oncogenes (such as CAV1), IncRNA, and miRNA were activated to promote cancer cell growth 
adjacent non-tumorous tissues from our tissue bank were used for IHC staining. The IHC analysis was conducted by using the DAB peroxidase substrate kit (Vector Lab, SK-4100) according to the protocol provided by the manufacturer. To analyze the distribution and expression level of ADI1, a homemade rabbit polyclonal antibody was used. The generation of rabbit polyclonal anti-ADI1 antibody was described in our previously study $^{6}$. A mouse monoclonal anti-CAV1 antibody (Proteintech, 66067-1-lg) was used for staining of Caveolin-1. The dilution of these two antibodies used in IHC was 1:200.

\section{Lysate preparation and western blot}

Protein extraction and western blot analysis were conducted as described previously ${ }^{41}$. The same amount of an aliquot of commercialized primary hepatocyte cell lysate was loaded into each gel as an additional batch-to-batch control and a quantification-referencing indicator (Supplementary Fig. S1A). Antibodies used for detection of ADI1 and Caveolin-1 in western blotting were the same as described in IHC staining in a dilution of 1:2000 and 1:5000, respectively. For detection of ATCB, a rabbit polyclonal antibody (Proteintech, 20536-1-AP) was used with 5000 -fold diluted.

\section{Lentivirus-mediated down-regulation of ADI1}

The lentivirus carrying ADI1 shRNA clones were purchased from Academia Sinica RNAi core facility, Taiwan. The lentivirus transduction was conducted as described previously $^{42}$. The clone IDs of ADI1 shRNAs used in this study were TRCN0000290230 for \#1 and TRCN0000296534 for \#2. The target sequence of these two clones were 5'-GAACTACTCCTGGATGGACAT-3' and $5^{\prime}$-ACTAACACGTGCCTCGTAAAG-3' for \#1 and $\# 2$, respectively. For complementary experiments, the cells used for transient transfection of empty vector or ADI1expressing plasmid were puromycin selected $(3-7 \mu \mathrm{g} / \mathrm{mL})$ and shRNAs stably expressed.

\section{Cell culture}

The cells used in this study, J7 and Huh7, were cultured in Dulbecco's modified Eagle's medium in a standard culture condition with $37^{\circ}$ and $5 \% \mathrm{CO}_{2}$ humidified incubator. For stable expression of interested genes or shRNAs, the antibiotic selection was utilized. The cells stably expressing ADI1 were maintained under the presence of $1 \mathrm{mg} / \mathrm{mL}$ of G418. The stably expressed ADI1 shRNA were selected by puromycin at the final concentration of $3-7 \mu \mathrm{g} / \mathrm{mL}$.

\section{Cell proliferation assay}

The cell proliferation rate was assessed as previously described with minor modifications ${ }^{43}$. Briefly, the alamar blue was used to replace Thiazolyl Blue Tetrazolium Bromide (MTT) for the lower cytotoxicity and simplicity.

\section{Plasmid constructs for ADI1 and CAV1 expression}

The DNA oligos listed below were used for ADI1 and CAV1 open reading frame amplification. The underlined sequences indicated the recognition sites for HindIII and $E c o$ RI in primer pair for ADI1, while for EcoRI and NotI in that for CAV1. Amplicons of wild type or mutant ADI1 and CAV1 were then inserted into pcDNA3.1/V5-HisB mammalian expression vector (Invitrogen, V81020) by using specific restriction sites. All the constructs used in this study were sequence verified by an automatic sequencer.

ADI1 Forward: 5'-AGTTAAGCTTATGGTGCAGGCC TGGTATAT-3'

ADI1 Reverse: 5'-TGCAGAATTCTTAGGCGGTCTG TGCCAGAA-3'

CAV1 Forward: 5'-ATCGGAATTCATGTCTGGGGG CAAATACGT-3'

CAV1 Reverse: 5'-AATTGCGGCCGCCTATATTTCT TTCTGCAAGTTGA-3'

\section{Colony formation assay}

To assess the capability of cell reproduction, the colony formation assay was conducted. The procedure was executed as described in our previous study ${ }^{42}$.

\section{TUNEL assay}

The cells stably expressing control vector or ADI1 were seeded to a 24-well plate with coverslip in the bottom. After $48-72 \mathrm{~h}$, the apoptosis status was determined by TUNEL assay as described previously ${ }^{42}$.

\section{Xenograft model}

The xenograft model was executed under the approval of Chang Gung Institutional Animal Care and Use Committee. Three-week-old BALB/c nude mice purchased from the National Laboratory Animal Center (Taipei, Taiwan) were used. The procedure was described previously $^{42}$.

\section{CDNA microarray}

The cDNA microarray was used for detection of genes mRNA levels and was conducted as previously described $^{44}$.

\section{RNA isolation and quantitative RT-PCR}

The RNA extraction was performed as previously described $^{45}$. The ToolScript MMLV RTase (TOOLS, TGERA04) was utilized for reverse transcription. Prior to RNA subjected into reverse transcription, the DNase digestion was conducted by using Turbo DNase I (Ambion, AM1907). Ten micrograms of total RNA was 
used for each digestion experiment. The detail procedures were accordance with the standard protocol provided by the manufacturers. Subsequently, $5 \mu \mathrm{g}$ of DNA digested RNAs were subjected into RT reaction by using ToolScript MMLV RTase (TOOLS, TGERA04). For each 20 $\mu \mathrm{L}$ reaction, $5 \mu \mathrm{L}$ of 50 -fold diluted cDNAs were utilized in RT-qPCR analysis. For quantitation of miRNAs, the tailing reverse transcription was employed as previously described $^{41}$. The Bio-rad CFX96 real time system was used for detection of interested genes. The DNA oligos and primer pairs used for examining gene expression in this study are listed in Supplementary Table S2.

\section{SAMe detection}

For assessment of SAMe levels, the Bridge-It ${ }^{\circledast} S$-Adenosyl Methionine (SAM) Fluorescence Assay Kit was used according to the standard procedures provided by the manufacturer. In Fig. 5A, the cells stably expressing scramble shRNA or ADI1 shRNA were used for transient expression of empty vector or ADI1-overexpressing plasmid. In Fig. 5b, the cells were only transiently expressing empty vector, wild type, and mutant ADI1s as indicated.

\section{DNMT inhibition experiment}

For inhibition of DNMT activity, the inhibitor, SGI1027, was employed ${ }^{46}$. At 24 or $48 \mathrm{~h}$ after transfection, 2 $\mu \mathrm{M}$ of this compound was supplemented in culture medium for $16-24 \mathrm{~h}$. The cells were then washed and collected for subsequent protein extraction or total RNA isolation.

\section{Analysis of DNA methylation status}

The CAV1 promoter was amplified as previously described $^{18}$. The EpiTect Bisulfite kit (QIAGEN, 59104) was used to convert genomic DNA. The experiment was executed according to the standard protocol provided by the manufacturer. The amplicon was purified and then subjected into next-generation sequencing (NGS) and examined the methylated cytosine in $C A V 1$ promoter as described previously ${ }^{47}$. The methyl-seq analysis was employed for examining whole-genome methylation status and executed by subjecting $2 \mu \mathrm{g}$ genomic DNA from either empty vector or ADI1-overexpressed J7 cells to TOOLS Co., Ltd. Analysis of sites with significant hyperor hypo-methylation was conducted by using specific filters, such as the level of methylation either in Empty vector or in the ADI1 OE group could not be "0", the difference of methylation level between these two groups should be more than 0.4 and the $q$-value (adjusted $p$ value) should be smaller than 0.001 . Only those genes with significant methylation status changes were candidates for further validation.

\section{Acknowledgements}

The authors thank the technicians in Liver Research Center, Chang Gung Memorial Hospital, Linkou branch, for their excellent technical help. This study was supported by grants from Chang Gung Memorial Hospital, Linkou branch, Taiwan (CRRPG3F0052, CMRPG3F1602).

\section{Authors' contributions}

Study concept and design: Y.-D.C., L.-M.P. and C.-T.Y. Execution of experiments: Y.-D.C., H.-Y.L., L.-M.P. and Y.-H.H. Statistical analysis and interpretation of data: Y.-D.C., Y.-H.H., Y.-H.L. and K.-H.L. Drafting of the manuscript: Y.-D.C., Y.-H.L. and C.-T.Y. The supplementary information is available at Cell Death and Disease's website. All authors read and approved the final manuscript.

\section{Author details \\ ${ }^{1}$ Liver Research Center, Chang Gung Memorial Hospital, Taoyuan, Taiwan. ${ }^{2}$ Molecular Medicine Research Center, Chang Gung University, Taoyuan, Taiwan. ${ }^{3}$ Department of Biochemistry, Chang Gung University, Taoyuan City, Taiwan. ${ }^{4}$ Graduate Institute of Biomedical Sciences, College of Medicine, Chang Gung University, Taoyuan City, Taiwan. ${ }^{5}$ Medical Research Department, Taipei Veterans General Hospital, Taipei, Taiwan}

Conflict of interest

The authors declare that they have no conflict of interest.

\section{Publisher's note}

Springer Nature remains neutral with regard to jurisdictional claims in published maps and institutional affiliations.

Supplementary Information accompanies this paper at (https://doi.org/ 10.1038/s41419-019-1486-4).

Received: 1 November 2018 Revised: 18 February 2019 Accepted: 25 February 2019

Published online: 11 March 2019

References

1. Siegel, R. L., Miller, K. D. \& Jemal, A. Cancer statistics, 2016. CA Cancer J. Clin. 66 7-30 (2016).

2. Clark, T., Maximin, S., Meier, J., Pokharel, S. \& Bhargava, P. Hepatocellular carcinoma: review of epidemiology, screening, imaging diagnosis, response assessment, and treatment. Curr. Probl. Diagn. Radiol. 44, 479-486 (2015).

3. Pang, T. C. \& Lam, V. W. Surgical management of hepatocellular carcinoma. World J. Hepatol. 7, 245-252 (2015).

4. Pascual, S., Herrera, I. \& Irurzun, J. New advances in hepatocellular carcinoma. World J. Hepatol. 8, 421-438 (2016)

5. Yeh, C. T., Lai, H. Y., Chen, T. C., Chu, C. M. \& Liaw, Y. F. Identification of a hepatic factor capable of supporting hepatitis $C$ virus replication in a nonpermissive cell line. J. Virol. 75, 11017-11024 (2001).

6. Cheng, J. C., Yeh, Y. J., Pai, L. M., Chang, M. L. \& Yeh, C. T. 293 cells overexpressing human ADI1 and CD81 are permissive for serum-derived hepatitis C virus infection. J. Med. Virol. 81, 1560-1568 (2009).

7. Chang, M. L., Huang, Y. H., Cheng, J. C. \& Yeh, C. T. Interaction between hepatic membrane type 1 matrix metalloproteinase and acireductone dioxygenase 1 regulates hepatitis C virus infection. J. Viral Hepat. 23, 256-266 (2016).

8. Dunwell, J. M., Purvis, A. \& Khuri, S. Cupins: the most functionally diverse protein superfamily? Phytochemistry 65, 7-17 (2004).

9. Chou, H. Y. et al. ADI1, a methionine salvage pathway enzyme, is required for Drosophila fecundity. J. Biomed. Sci. 21, 64 (2014).

10. Pirkov, I., Norbeck, J., Gustafsson, L. \& Albers, E. A complete inventory of all enzymes in the eukaryotic methionine salvage pathway. FEBS J. $\mathbf{2 7 5}$ 4111-4120 (2008)

11. Uekita, T. et al. Membrane-type 1 matrix metalloproteinase cytoplasmic tailbinding protein-1 is a new member of the Cupin superfamily. A possible multifunctional protein acting as an invasion suppressor down-regulated in tumors. J. Biol. Chem. 279, 12734-12743 (2004). 
12. Hirano, W., Gotoh, I., Uekita, T. \& Seiki, M. Membrane-type 1 matrix metalloproteinase cytoplasmic tail binding protein-1 (MTCBP-1) acts as an eukaryotic aci-reductone dioxygenase (ARD) in the methionine salvage pathway. Genes Cells 10, 565-574 (2005).

13. Deshpande, A. R., Pochapsky, T. C., Petsko, G. A. \& Ringe, D. Dual chemistry catalyzed by human acireductone dioxygenase. Protein Eng. Des. Sel. 30 , 197-204 (2017)

14. Oram, S. W. et al. Expression and function of the human androgen-responsive gene AD11 in prostate cancer. Neoplasia 9, 643-651 (2007).

15. Pratt, J., Iddir, M., Bourgault, S. \& Annabi, B. Evidence of MTCBP-1 interaction with the cytoplasmic domain of MT1-MMP: implications in the autophagy cell index of high-grade glioblastoma. Mol. Carcinog. 55, 148-160 (2016).

16. Tang, B., Kadariya, Y., Murphy, M. E. \& Kruger, W. D. The methionine salvage pathway compound 4-methylthio-2-oxobutanate causes apoptosis independent of down-regulation of ornithine decarboxylase. Biochem. Pharmacol. 72, 806-815 (2006).

17. Fernandez-Rojo, M. A. \& Ramm, G. A. Caveolin-1 function in liver physiology and disease. Trends Mol. Med. 22, 889-904 (2016).

18. Hirasawa, $Y$. et al. Methylation status of genes upregulated by demethylating agent 5-aza-2'-deoxycytidine in hepatocellular carcinoma. Oncology 71, 77-85 (2006).

19. Li, S. et al. The long non-coding RNA TP73-AS1 modulates HCC cell proliferation through miR-200a-dependent HMGB1/RAGE regulation. J. Exp. Clin. Cancer Res. 36, 51 (2017).

20. Sun, C. C. et al. Long intergenic noncoding RNA 00511 acts as an oncogene in non-small-cell lung cancer by binding to EZH2 and suppressing p57. Mol. Ther. Nucleic Acids 5, e385 (2016).

21. He, D. et al. MiR-371-5p facilitates pancreatic cancer cell proliferation and decreases patient survival. PLoS ONE 9, e112930 (2014).

22. Liu, R. Y. et al. miR-371-5p down-regulates pre mRNA processing factor 4 homolog B (PRPF4B) and facilitates the G1/S transition in human hepatocellular carcinoma cells. Cancer Lett. 335, 351-360 (2013).

23. Shahabi, A. et al. Novel gene expression signature predictive of clinical recurrence after radical prostatectomy in early stage prostate cancer patients. Prostate 76, 1239-1256 (2016).

24. Ansorena, E. et al. S-adenosylmethionine and methylthioadenosine are antiapoptotic in cultured rat hepatocytes but proapoptotic in human hepatoma cells. Hepatology 35, 274-280 (2002).

25. Lu, S. C. \& Mato, J. M. S-Adenosylmethionine in cell growth, apoptosis and liver cancer. J. Gastroenterol. Hepatol. 23, S73-S77 (2008).

26. Bistul, G. et al. The essential role of methylthioadenosine phosphorylase in prostate cancer. Oncotarget 7, 14380-14393 (2016).

27. Hong, S. H. et al. APIP, an ERBB3-binding partner, stimulates erbB2-3 heterodimer formation to promote tumorigenesis. Oncotarget 7, 21601-21617 (2016).

28. Benavides, M. A. et al. L-Methionine inhibits growth of human pancreatic cancer cells. Anticancer Drugs 25, 200-203 (2014).

29. Kang, W. et al. Structural and biochemical basis for the inhibition of cell death by APIP, a methionine salvage enzyme. Proc. Natl Acad. Sci. USA 111, E54-E61 (2014).
30. Frau, M., Feo, F. \& Pascale, R. M. Pleiotropic effects of methionine adenosyltransferases deregulation as determinants of liver cancer progression and prognosis. J. Hepatol. 59, 830-841 (2013).

31. Yang, $\mathrm{H}$. et al. S-adenosylmethionine and its metabolite induce apoptosis in HepG2 cells: Role of protein phosphatase 1 and $\mathrm{BCl}-\mathrm{x}(\mathrm{S})$. Hepatology 40, 221-231 (2004)

32. Garcea, R. et al. Variations of ornithine decarboxylase activity and S-adenosyl-Lmethionine and 5'-methylthioadenosine contents during the development of diethylnitrosamine-induced liver hyperplastic nodules and hepatocellular carcinoma. Carcinogenesis 8, 653-658 (1987).

33. Pascale, R. M. et al. Chemoprevention of rat liver carcinogenesis by S-adenosylL-methionine: a long-term study. Cancer Res. 52, 4979-4986 (1992).

34. Lu, S. C. et al. S-adenosylmethionine in the chemoprevention and treatment of hepatocellular carcinoma in a rat model. Hepatology 50, 462-471 (2009).

35. Lu, S. C. \& Mato, J. M. Role of methionine adenosyltransferase and Sadenosylmethionine in alcohol-associated liver cancer. Alcohol 35, 227-234 (2005).

36. Anstee, Q. M. \& Day, C. P. S-adenosylmethionine (SAMe) therapy in liver disease: a review of current evidence and clinical utility. J. Hepatol. 57, 1097-1109 (2012).

37. Morgan, T. R. et al. A phase \| randomized, controlled trial of Sadenosylmethionine in reducing serum alpha-fetoprotein in patients with hepatitis C cirrhosis and elevated AFP. Cancer Prev. Res. 8, 864-872 (2015).

38. Guo, T., He, Y., Ma, W., Liu, Z. \& Liu, Q. Feasibility and efficacy of S-adenosyl-Lmethionine in patients with HBV-related HCC with different BCLC stages. Gastroenterol. Res. Pract. 2016, 4134053 (2016)

39. Bottiglieri, T. S-Adenosyl-L-methionine (SAMe): from the bench to the bedside - molecular basis of a pleiotrophic molecule. Am. J. Clin. Nutr. 76, 1151S-1157S (2002)

40. Meyer, K. D. \& Jaffrey, S. R. The dynamic epitranscriptome: N6 methyladenosine and gene expression control. Nat. Rev. Mol. Cell Biol. 15 313-326 (2014).

41. Chu, Y. D. et al. RACK-1 regulates let-7 microRNA expression and terminal cell differentiation in Caenorhabditis elegans. Cell Cycle 13, 1995-2009 (2014).

42. Huang, Y. H. et al. HBV polymerase overexpression due to large core gene deletion enhances hepatoma cell growth by binding inhibition of microRNA100. Oncotarget 7, 9448-9461 (2016).

43. Huang, Y. H. et al. Identification of postoperative prognostic microRNA predictors in hepatocellular carcinoma. PLOS ONE 7, e37188 (2012).

44. Lai, M. W. et al. Hepatocarcinogenesis in transgenic mice carrying hepatitis $B$ virus pre-S/S gene with the SW172* mutation. Oncogenesis 5, e273 (2016).

45. Chu, Y. D., Chen, H. K., Huang, T. \& Chan, S. P. A novel function for the DEADbox RNA helicase DDX-23 in primary microRNA processing in Caenorhabditis elegans. Dev. Biol. 409, 459-472 (2016).

46. Datta, J. et al. A new class of quinoline-based DNA hypomethylating agents reactivates tumor suppressor genes by blocking DNA methyltransferase 1 activity and inducing its degradation. Cancer Res. 69, 4277-4285 (2009).

47. Liang, K. H. et al. Peginterferon is superior to nucleos(t)ide analogues for prevention of hepatocellular carcinoma in chronic hepatitis B. J. Infect. Dis. 213 966-974 (2016) 